\title{
On intersective polynomials with non-solvable Galois group
}

\author{
J. König*
}

July 30, 2018

\begin{abstract}
We present new theoretical results on the existence of intersective polynomials (that is, integer polynomials with roots in all $\mathbb{Q}_{p}$, but not in $\mathbb{Q}$ ) with certain prescribed Galois groups, namely the projective and affine linear groups $P G L_{2}(\ell)$ and $A G L_{2}(\ell)$ as well as the affine symplectic groups $A G S p_{4}(\ell):=$ $\left(\mathbb{F}_{\ell}\right)^{4} \rtimes G S p_{4}(\ell)$. For further families of affine groups, existence results are proven conditional on the existence on certain tamely ramified Galois extensions. We also compute explicit families of intersective polynomials for certain non-solvable groups.
\end{abstract}

Keywords: Galois theory; polynomials; number fields; group theory; Galois representations.

\section{Introduction}

Let $f \in \mathbb{Z}[X]$ be a monic polnomial with integer coefficients. $f$ is called intersective, if $f$ has a root modulo every $n \in \mathbb{N}$. Equivalently, $f$ has a root in $\mathbb{Q}_{p}$ for every prime $p$. $f$ is called non-trivially intersective, if $f$ does not have a rational root.

A simple example of a non-trivially intersective polynomial is $\left(X^{2}-2\right)\left(X^{2}+7\right)\left(X^{2}+14\right)$.

In the following, "intersective" should always be understood as "non-trivially intersective". The existence of intersective polynomials with a given number of irreducible factors and a given Galois group $G$ is linked closely to the following property of the group $G$.

*Universität Würzburg, Emil-Fischer-Str. $\quad 30,97074 \quad$ Würzburg, $\quad$ Germany. $\quad$ email: 


\section{Introduction}

Definition 1.1 ( $k$-coverable group). Let $G$ be a finite group and $k \in \mathbb{N}$. $G$ is called $k$ coverable if there exist proper subgroups $U_{1}, \ldots, U_{k}$ of $G$ such that every element of $G$ is contained in a conjugate of one of the $U_{i}$, in other words if

$$
G=\bigcup_{i=1}^{k} \bigcup_{x \in G} U_{i}^{x} .
$$

An easy exercise in undergraduate algebra shows that there are no 1-coverable finite groups (and therefore, by the following exposition, there are no irreducible intersective polynomials). Several authors have studied 2-coverable groups (cf. e.g. [3], 4] or [17]).

It is easy to verify that if, for a given group $G$, there is an intersective polynomial with $k$ irreducible monic factors over $\mathbb{Z}$ and with Galois group $G$, then $G$ must be $k$-coverable. More precisely, let $f=f_{1} \cdots f_{k}$ be a complete factorization of the intersective polynomial $f$ over $\mathbb{Z}$. Let $E \mid \mathbb{Q}$ be the Galois closure of $f$, and let $p$ be a prime that does not ramify in $E$. Then the Galois group $G_{p}$ of the localization $E_{p} \mid \mathbb{Q}_{p}$ is cyclic and fixes one of the roots of some $f_{i}$, since by definition, $f$ has a root in $\mathbb{Q}_{p}$. This means that $G_{p}=:\langle\sigma\rangle$ is contained in a conjugate of the proper subgroup $U_{i}:=\operatorname{Gal}(E \mid \mathbb{Q}(\alpha))$, for a root $\alpha$ of $f_{i}$. On the other hand, by the Chebotarev density theorem, elements of every conjugacy class of $G$ occur as generators of such a decomposition group $G_{p}$. Therefore $G$ is the union of the conjugates of all $U_{i}(i=1, \ldots, k)$.

This means that, on the other hand, a given Galois extension $E \mid \mathbb{Q}$ with a $k$-coverable Galois group $G$ will yield intersective polynomials with $k$ irreducible factors, provided that the decomposition groups at the ramified primes in $E$ behave appropriately. More precisely, Sonn shows the following in Proposition 1 of [20]:

Proposition 1.1. Let $K \mid \mathbb{Q}$ be a Galois extension with Galois group $G$. Then the following are equivalent:

i) $K$ is the splitting field of a non-trivially intersective polynomial $f \in \mathbb{Z}[X]$ with exactly $k$ irreducible factors.

ii) $G$ is $k$-coverable by subgroups $U_{1}, \ldots, U_{k}$, the intersection of all conjugates $U_{i}^{g}$ (with $i=1, \ldots, k$ and $g \in G$ ) is trivial, and every decomposition group $D_{p}$ in $K($ for $p \in \mathbb{P})$ is contained in a conjugate of one of the $U_{i}$.

Note that the condition of trivial intersection in ii) of Prop. 1.1 is automatically satisfied if $G$ is simple, or if one of the $U_{i}$ is a point stabilizer in a faithful permutation action of $G$. As this will always be the case in our examples in the following sections, we therefore will 


\section{Existence results}

not mention this condition there.

In [21], Sonn shows that intersective polynomials exist for all non-solvable groups that occur as Galois groups over $\mathbb{Q}$. He also shows in [20, Theorem 2] that for solvable groups that admit a $k$-covering of subgroups with the trivial intersection property of Prop. 1.1ii), intersective polynomials with exactly $k$ irreducible factors exist 1 For most non-solvable groups (even the ones known to occur as Galois groups over $\mathbb{Q}$ ), this problem remains open:

Question 1: Let $G$ be a finite group, $k$-coverable by subgroups $U_{1}, \ldots, U_{k}$ such that the intersection of all conjugates $U_{i}^{g}$ (with $i=1, \ldots, k$ and $g \in G$ ) is trivial. Does there exist an intersective polynomial $f=f_{1} \cdots f_{k} \in \mathbb{Z}[X]$ with exactly $k$ irreducible factors and with Galois group $G$ ?

In this paper, we focus on 2-coverable groups. Intersective polynomials have been given for the 2-coverable alternating and symmetric groups ([17]) as well as for the dihedral group $D_{5}([10])$, and their existence has been shown for non-solvable Frobenius groups ([20]). Using results from the theory of Galois representations, we will derive the existence of intersective polynomials with two irreducible factors for several infinite families of groups, namely the groups $P G L_{2}(\ell)$ and $A G L_{2}(\ell)$ as well as the affine symplectic groups $A G S p_{4}(\ell)$ with an arbitrary prime $\ell$.

For small 2-coverable groups, one can nowadays easily check the databases of number fields ([7] or [8]) to find fields with suitable ramification conditions, yielding examples of intersective polynomials with two irreducible factors for the prescribed group. A somewhat more challenging task is to find nice parametric families for a given group. We give examples of such families for several non-solvable groups in Section 3 ,

\section{Existence results}

We briefly recall some basic facts about decomposition groups and inertia groups:

Lemma 2.1. Let $E \mid \mathbb{Q}$ be a Galois extension, $p$ be a prime and $E_{p} \mid \mathbb{Q}_{p}$ be the localization at $p$. Let $D_{p}, I_{p}$ and $I_{p, w}$ denote the decomposition group, the inertia group and the wild inertia group at a prime ideal of $E$ extending $p$. Then $I_{p, w} \unlhd I_{p} \unlhd D_{p}$ is a chain of normal subgroups of $D_{p}$, the quotient $D_{p} / I_{p}$ is cyclic, the quotient $I_{p} / I_{p, w}$ is cyclic of order coprime to $p$, and $I_{p, w}$ is a p-group. In particular $D_{p}$ is solvable.

\footnotetext{
${ }^{1}$ As noted in [20, Theorem 2], such a $k$-covering is possible (for some $k$ ) for all non-cyclic groups.
} 


\section{Existence results}

\subsection{The groups $P G L_{2}(\ell)$}

The groups $P G L_{2}(\ell)$, with $\ell$ a prime, are all known to occur as Galois groups over $\mathbb{Q}$, thanks to the theory of ordinary Galois representations. In this section we show how such representations can be used to obtain intersective polynomials with two irreducible factors in $\mathbb{Z}[X]$, and with Galois group $P G L_{2}(\ell)$. In [4], it is shown that for any prime power $q$, $P G L_{n}(q)$ is 2-coverable if and only if $2 \leq n \leq 4$.

In particular, for $n=2$, one has the following.

Theorem 2.2. Let $q \geq 4$ be a prime power. Let $U_{1}$ be a point stabilizer of $P G L_{2}(q)$ in its natural degree $q+1$ action on 1-dimensional subspaces. Let $U_{2}$ be the normalizer of a Singer cycle (i.e., of a cyclic subgroup of order $q+1$ ) in $P G L_{2}(q)$. Then $U_{1}$ and $U_{2}$ yield a 2-covering of $P G L_{2}(q)$.

From now on, let $\ell \geq 5$ be a prime. To ensure the existence of a pair $(f, g)$ with $f, g \in \mathbb{Z}[X]$ monic and irreducible, such that $G a l(f \cdot g) \cong P G L_{2}(\ell)$ and $f \cdot g$ has a root in every $\mathbb{Q}_{p}$, we need a $P G L_{2}(\ell)$-extension $K \mid \mathbb{Q}$ such that all decomposition groups $\operatorname{Gal}\left(K_{p} \mid \mathbb{Q}_{p}\right)$ are contained either in a point stabilizer or in the normalizer of a singer cycle. One way to do this is to make use of results on tamely ramified extensions, as presented in [1]. We briefly summarize the construction given there. Cf. especially the proof of [1, Thm. 2.1].

For every prime $\ell \geq 5$, there exists a semistable elliptic curve with good supersingular reduction at $\ell$, such that the attached Galois representation $\rho: G a l_{\mathbb{Q}} \rightarrow G L_{2}\left(\mathbb{F}_{\ell}\right)$ is surjective (the surjectivity follows from the semistability for all $\ell \geq 11$ by a theorem of Mazur ([14, Theorem 4]); and the smaller $\ell$ are treated explicitly in [1]). Composition of this representation with the projection $G L_{2}\left(\mathbb{F}_{\ell}\right) \rightarrow P G L_{2}\left(\mathbb{F}_{\ell}\right)$ yields a Galois extension of $\mathbb{Q}$ with group $P G L_{2}\left(\mathbb{F}_{\ell}\right)$.

Because of semistability, the inertia group at every prime $p \neq \ell$ is either trivial or of order $\ell$. In particular, all decomposition groups at the ramified primes $p \neq \ell$ are contained in the normalizer of an $\ell$-Sylow subgroup of $P G L_{2}(\ell)$, i.e. a conjugate of $U_{1}$.

Furthermore, because of good supersingular reduction at $\ell$, the inertia subgroups at $\ell$ are cyclic of order $\ell+1$ (i.e., Singer cycles), and therefore the decomposition subgroups are contained in a conjugate of $U_{2}$. This follows from Proposition 12 in [18].

We therefore obtain:

Theorem 2.3. For every prime $\ell \geq 5$, there is an intersective polynomial with two monic irreducible factors $f, g \in \mathbb{Z}[X]$ such that $\operatorname{Gal}(f g \mid \mathbb{Q})=P G L_{2}(\ell)$. 


\section{Existence results}

\subsection{The groups $A G L_{2}(\ell)$}

For $\ell \geq 5$ a prime, we will deduce the existence of intersective polynomials with two irreducible factors for the affine linear groups $A G L_{2}(\ell)$ from the corresponding results for $P G L_{2}(\ell)$. First note that the existence of Galois extensions over $\mathbb{Q}$ with Galois group $A G L_{2}(\ell)$ follows immediately from the existence of those with group $G L_{2}(\ell)$, since any split embedding problem with abelian kernel (here, with kernel $\left.\left(C_{\ell}\right)^{2}\right)$ possesses a solution over $\mathbb{Q}$ (see Theorem IV.2.4 in [13]).

The groups $A G L_{n}(q)$ are 2-coverable even for arbitrary $n \geq 2$ and prime powers $q$.

Theorem 2.4. Let $q$ be a prime power, $n \geq 2$ and let $G:=A G L_{n}(q)$ be the affine linear group in its natural primitive action on $V:=\left(\mathbb{F}_{q}\right)^{n}$. Let $U_{1}$ be a point stabilizer and $U_{2}:=V \rtimes H$, where $H \leq G L_{n}(q)$ is a stabilizer of a one-dimensional subspace in $V$. Then $U_{1}$ and $U_{2}$ yield a 2-covering of $A G L_{n}(q)$.

Proof. Any element of $G$ is a map of the form $\varphi: x \mapsto A x+v$, with $v \in V, A \in G L_{n}(q)$. Now if $v$ is in the image of $I_{n}-A$ ( $I_{n}$ the identity matrix), then $\varphi$ fixes a point in $V$, and therefore (by the transitivity of the action on $V$ ) lies in a conjugate of $U_{1}$. But otherwise, $A$ must have an eigenvalue 1 , so it fixes a one-dimensional subspace and therefore lies in a conjugate of $H$. Therefore $\varphi$ lies in a conjugate of $V H$.

Now let $n=2$ and $q=\ell \geq 5$ be a prime.

First, assume $\ell \geq 5$. Let $E$ be a semistable elliptic curve over $\mathbb{Q}$ with ordinary good reduction at $\ell$. The theory of Galois representations yields an epimorphism a Galois extension $K \mid \mathbb{Q}$ with group $G L_{2}(\ell)$ and the following ramification (cf. the corollary after Proposition 11 in [18]):

i) At prime ideals of $K$ extending $\ell$, the inertia group either has order $\ell-1$ and is conjugate to the group of the form $\left(\begin{array}{ll}\star & 0 \\ 0 & 1\end{array}\right)$, or has order $\ell \cdot(\ell-1)$ and is conjugate to the group of the form $\left(\begin{array}{ll}\star & \star \\ 0 & 1\end{array}\right)$.

ii) At prime ideals extending a prime $p \neq \ell$, by semistability, the inertia group is either trivial or of order $\ell$, conjugate to a subgroup of $\left(\begin{array}{ll}1 & \star \\ 0 & 1\end{array}\right)$.

The normalizer of the first group in case i) is just the group of diagonal matrices in $G L_{2}(\ell)$, whereas the normalizers of the other cases contain a normal subgroup of order $\ell$. This shows 


\section{Existence results}

immediately that the decomposition group at any ramified prime is contained in a conjugate of the Borel subgroup (as these are the normalizers of an order- $\ell$ subgroup), which is just the stabilizer of a one-dimensional subspace in $V:=\left(\mathbb{F}_{\ell}\right)^{2}$. By [13, Theorem IV.2.4], there is an extension $K_{2} \supset K$ such that $K_{2} \mid \mathbb{Q}$ is Galois with group $A G L_{2}(\ell)$. The decomposition groups at all ramified primes in $K_{2} \mid \mathbb{Q}$ that extend primes already ramified in $K \mid \mathbb{Q}$ are then contained in a conjugate of $V H$, with $H$ the Borel subgroup in $G L_{2}(\ell)$. But for ramified primes of $K_{2} \mid \mathbb{Q}$ extending unramified primes of $K \mid \mathbb{Q}$ (note that in particular those primes must extend a rational prime $p \neq \ell$ ), the inertia subgroup must be cyclic of order $\ell$ and therefore the decomposition subgroup normalizes a 1-dimensional subspace of $V$, i.e. it is also contained in a conjugate of $V H$.

The cases $\ell \in\{5,7\}$ can be dealt with by explicit Galois realizations from the databases. Alternatively, they are covered by the results of the next section. Together with Theorem 2.4, we therefore obtain:

Theorem 2.5. For every prime $\ell \geq 5$, there is an intersective polynomial with two monic irreducible factors $f, g \in \mathbb{Z}[X]$ such that $G a l(f g \mid \mathbb{Q})=A G L_{2}(\ell)$.

\subsection{Further affine groups}

We will generalize the results of the previous section for further affine groups, conditional on the existence of certain tamely ramified Galois extensions of $\mathbb{Q}$. More precisely we will show:

Theorem 2.6. Let $V=\left(\mathbb{F}_{q}\right)^{n}$ a finite vector space of dimension $2 \leq n \leq 4$. Let $G=V \rtimes U$ be a finite affine group on $V$, with a group $U \leq G L(V)$ which acts transitively on the 1dimensional subspaces of $V$.

If $U$ occurs as a Galois group of a tamely ramified Galois extension over $\mathbb{Q}$, then there is an intersective polynomial $f \cdot g \in \mathbb{Z}[X]$ with two irreducible factors and with Galois group $G$.

The first important observation for a proof of Thm. 2.6 is that the 2-covering constructed for the full affine groups $A G L_{n}(q)$ in the previous section can be generalized in a natural way to affine groups $G=V \rtimes U$ (with arbitrary $n \geq 2, V:=\left(\mathbb{F}_{q}\right)^{n}$ and $U \leq G L_{n}\left(\mathbb{F}_{q}\right)$ transitive on the 1-dimensional subspaces). The proof is completely analogous to the one of Thm. 2.4 (note that the transitivity of $U$ is needed because in the proof of Thm. 2.4, the corresponding stabilizers in $U$ need to be conjugate).

Now for $n \leq 4$ we will show that even more holds: 


\section{Existence results}

Lemma 2.7. Let $V=\left(\mathbb{F}_{q}\right)^{n}$ a finite vector space of dimension $2 \leq n \leq 4$. Let $G=V \rtimes U$ be as in Theorem 2.6. Let $U_{1}$ be a point stabilizer and $U_{2}:=V \rtimes H$, where $H \leq U$ is a stabilizer of a one-dimensional subspace in $V$. Then every metacyclic subgroup of $G$ is contained in a conjugate of $U_{1}$ or of $U_{2}$.

Proof. Let $M \leq G$ be metacyclic, i.e. $M=\langle\phi, \psi\rangle$, with $\phi, \psi \in G$ such that $\psi$ normalizes $\langle\phi\rangle$. We will break the proof down into several cases. We always write $\phi: V \rightarrow V, x \mapsto A x+b$ and $\psi: x \mapsto C x+d$.

First, assume that $\phi$ fixes a point in $V$. By transitivity, we can assume that this fixed point is $0 \in V$, i.e. $\phi: x \rightarrow A x$ for some $A \in G L(V)$. Let $\psi$ be defined as $\psi: x \mapsto C x+d$ (with $d \in V, C \in G L(V))$. We will write $(d, C)$ for this map as an element of the semidirect product $G=V \rtimes U$. The normalizer condition then writes as

$$
(A(d), A C)=(0, A) \cdot(d, C)=(d, C) \cdot\left(0, A^{k}\right)=\left(d, C A^{k}\right)
$$

for some $k \in \mathbb{N}$. Therefore, $A$ fixes the vector $d$. Let $V_{0} \leq V$ be the subspace of elements fixed by $A$. Clearly, $\psi$ acts on $V_{0}$. If $\psi$ fixes a point in $V_{0}$ as well, then $\phi$ and $\psi$ lie in the same $G$-conjugate of $U_{1}$. But if $\psi$ acts without fixed point on $V_{0}$ (in particular, $V_{0} \neq\{0\}$ ), $C$ restricted to $V_{0}$ has an eigenvalue 1 (just as in the proof of Thm. 2.4). So $A$ and $C$ have a common fixed point, whence $\phi$ and $\psi$ lie in the same conjugate of $U_{2}$.

Next, assume that $\phi: x \rightarrow A x+b$ fixes no point in $V$ (in particular, $A$ has an eigenvalue 1 ), and that the minimal polynomial of $A$ is divisible by $X-1$ exactly once. Then $V=$ $E_{1} \oplus\left(I_{n}-A\right)(V)$, with $E_{1}$ the eigenspace of $A$ with eigenvalue 1 . By conjugating with an appropriate element $\left(v, I_{n}\right) \in A G L(V)$, we can translate $b$ by any element of $\left(I_{n}-A\right)(V)$, so we may assume $b \in E_{1}$. Then the normalizer condition reduces to

$$
\underbrace{(b, A)(d, C)}_{=(b+A(d), \ldots)}=\underbrace{(d, C)\left(k \cdot b, A^{k}\right)}_{=(d+k C(b), \ldots)}
$$

for some $k \in \mathbb{N}$. Therefore $\left(I_{n}-A\right)(d)=b-k C(b)$, and since $C$ fixes the eigenspace $E_{1}$ of $A$ setwise, this vector lies in $\left(I_{n}-A\right)(V) \cap E_{1}=\{0\}$. Therefore $b=k C(b)$, which enforces either $b=0$ (contradicting the assumption that $\phi$ is fixed point free) or $b$ is a common eigenvector of $A$ and $C$. Therefore $\phi$ and $\psi$ lie in the same conjugate of $U_{2}$.

Note that so far, we have not used the assumption $n \leq 4$.

Lastly, assume that the minimal polynomial of $A$ is divisible by $(X-1)^{2}$. Now if $A$ has an eigenvalue with geometric multiplicity one or a unique Jordan block of maximal size then 


\section{Existence results}

it follows immediately that $A$ and $C$ have a common eigenvector (since $C$, normalizing $\langle A\rangle$, leaves all higher eigenspaces $\operatorname{ker}\left(\lambda I_{n}-A\right)^{r}$ invariant). But as $n \geq 4$, this only leaves one possibility for $A$ up to conjugation in $G L(V)$, namely the Jordan form $A=\left(\begin{array}{rrrr}1 & 1 & & \\ 0 & 1 & & \\ & & 1 & 1 \\ & 0 & 1\end{array}\right)$.

One verifies by an easy calculation that via conjugating $(b, A)$ with appropriate elements $(v, M)$, with $M \in C_{G L(V)}(A)$, we can additionally assume $b=(0,0,0,1)^{t}=: e_{4}$.

The condition $\psi^{-1} \varphi \psi=\varphi^{k}$ (for some $k \in \mathbb{N}$ coprime to $\operatorname{ord}(A)=\operatorname{char}\left(\mathbb{F}_{q}\right)$ ) then reduces to

$$
C^{-1} A C=A^{k}
$$

and

$$
b-(I-A)(d)=C(\underbrace{\sum_{j=0}^{k-1} A^{j} b}_{=k \cdot e_{4}+\lambda e_{3} \text { for some } \lambda}) .
$$

Since $b-(I-A)(d) \in\left\langle e_{1}, e_{3}, e_{4}\right\rangle$ and anyway $C e_{3} \in \operatorname{ker}(I-A)=\left\langle e_{1}, e_{3}\right\rangle$, it follows that $C e_{4} \in\left\langle e_{1}, e_{3}, e_{4}\right\rangle$. Therefore $C e_{3}=C(A-I) e_{4}=\left(A^{m}-I\right) C e_{4}$ (with $m$ the multiplicative inverse of $k$ modulo ord $(A))$, and this lies in $\left(\sum_{j=0}^{m-1} A^{j}\right)(A-I)\left\langle e_{1}, e_{3}, e_{4}\right\rangle=$ $\left(\sum_{j=0}^{m-1} A^{j}\right)\left\langle e_{3}\right\rangle=\left\langle e_{3}\right\rangle$. We have therefore shown that $e_{3}$ is a common eigenvector for $A$ and $C$.

This concludes the proof.

We are now ready to prove Theorem 2.6. In the proof, we will use results on solvability of split embedding problems with nilpotent kernel with extra conditions on ramification, presented in [16, Thm. 9.5.11] as part of the proof of Shafarevich's theorem. This approach was also used by Sonn in [20] to show the existence of intersective polynomials for solvable groups and Frobenius groups.

Proof of Thm. 2.6. Let $2 \leq n \leq 4$, let $G=V \rtimes U$ and let $K \mid \mathbb{Q}$ be a tamely ramified Galois extension with group $U$. By [13, Theorem IV.2.4], there is an extension $L \mid K$ with $\operatorname{Gal}(L \mid \mathbb{Q})=G$ (split embedding problem with abelian kernel). Furthermore, by [16, Thm. 9.5.11], $L$ can be chosen such that every ramified prime of $K \mid \mathbb{Q}$ splits completely in $L \mid K$, and every prime of $K$ that ramifies in $L$ is completely split in $K \mid \mathbb{Q}$. Therefore all decomposition groups of ramified primes in $L \mid \mathbb{Q}$ either lie in $V \subset U_{2}$ (for primes extending a completely split prime of $K \mid \mathbb{Q}$ ); or they are metacyclic and intersect $V$ trivially (for primes that are completely split in $L \mid K)$, in which case we have just shown in Lemma 2.7 that 


\section{Existence results}

they lie in a conjugate of either $U_{1}$ or $U_{2}$ (here, $U_{i}$ are as in Lemma 2.7). This shows the assertion.

Remark: The groups in Lemma 2.7 include in particular the doubly transitive affine groups (of the corresponding dimensions). These groups are completely classified due to work of Hering $([6])$. They are of the form $G=V \rtimes U$ with $V=\left(\mathbb{F}_{p}\right)^{n}$ and one of the following:

a) $U \leq \Gamma L_{1}\left(p^{n}\right)$

b) $U \unrhd S L_{a}(q)$, with $q^{a}=p^{n}$,

c) $U \unrhd S p_{2 a}(q)$, with $q^{2 a}=p^{n}$,

d) $U \unrhd G_{2}^{\prime}(q)$, with $p^{n}=2^{n}=q^{6}$,

e) $(V, U)$ in an explicitly known finite list of "sporadic" cases.

The groups in a) are solvable and have therefore already been dealt with in [20]. The groups in b) include the case $U=G L_{2}(\ell)$ for which tamely ramified extensions are known (cf. Section 2.1); we therefore regain the statement of Theorem 2.5 as a special case of Theorem 2.6. However, the proof in Section 2.2 still has its own merit as it works for any solution of the $A G L_{2}(\ell)$-embedding problem beginning from the given $G L_{2}(\ell)$-extension, whereas Theorem 2.6 requires very speficic solutions for this embedding problem.

The groups in c) include in particular the groups of symplectic similitudes $G \operatorname{Sp}_{4}(\ell)$ for primes $\ell$. For these groups, the existence of tamely ramified Galois extensions of $\mathbb{Q}$ is known, again by work of Arias-de-Reyna and Vila ([2]). This immediately yields:

Corollary 2.8. Let $\ell$ be a prime. Then the affine symplectic group $A G S p_{4}(\ell)=\left(\mathbb{F}_{\ell}\right)^{4} \rtimes$ $G S p_{4}(\ell)$ occurs as the Galois group of an intersective polynomial $f \cdot g \in \mathbb{Z}[X]$ with exactly two irreducible factors.

Question: Can the assumption $n \leq 4$ in Lemma 2.7 be dropped? The only critical case is for unipotent matrices as in the last case of the proof of Lemma 2.7. While for $n \geq 5$, it is in general no longer true that the whole normalizer of such elements in $A G L_{n}(q)$ has a common fixed point in the coset action on $G / U_{2}$, computional evidence suggests that the assertion remains true for metacyclic subgroups. 
3 New explicit intersective polynomials for non-solvable groups

\section{New explicit intersective polynomials for non-solvable groups}

We present explicit infinite families of intersective polynomials with two irreducible factors for several non-solvable groups. Note that all of our families have a positive density subset of integer specializations of the extra parameter(s) yielding intersective polynomials. On the other hand, by Hilbert's irreducibility theorem, the set of integer specializations that do not preserve the Galois group has density 0. Therefore, one indeed obtains infinitely many intersective polynomials with the desired Galois group.

To prove that a given polynomial is actually intersective, the following well-known lemma is useful. It connects the splitting behaviour of the mod- $p$ reduction of a polynomial with the orbits of the inertia and decomposition subgroups at primes extending $p$.

Lemma 3.1. Let $f \in \mathbb{Z}[X]$ be monic and irreducible, let $p$ be a prime and $D_{p}$ resp. $I_{p}$ be the decomposition group resp. the inertia group of a prime extending $p$ in a splitting field of $f$, viewed as a permutation group on the zeroes of $f$. Assume that $f$ splits as $f \equiv \prod_{i=1}^{r} f_{i}^{k_{i}}$ modulo $p$. Then the orbit lengths of $D_{p}$ form a subpartition of the partition $\left[n_{1} \cdot k_{1}, \ldots, n_{r} \cdot k_{r}\right]$, where $n_{i}:=\operatorname{deg}\left(f_{i}\right)$. Similarly, the orbit lengths of $I_{p}$ form a subpartition of $[\underbrace{k_{1}, \ldots, k_{1}}_{n_{1} \text { times }}, \ldots, k_{r}, \ldots, k_{r}]$.

Proof. The orbit lengths of $D_{p}$ are exactly the degrees of the irreducible factors of $f$ over $\mathbb{Q}_{p}$. Let $g$ be such a factor. By Hensel's Lemma (cf. e.g. Lemma II.4.6 in [15]), the mod- $p$ reduction of $g$ can only have one irreducible factor (up to multiplicity). This shows the assertion about $D_{p}$. The claim about $I_{p}$ follows analogously after replacing $\mathbb{Q}_{p}$ by its maximal unramified extension.

This lemma will help to exclude certain ramification patterns for polynomials in an explicitly given family.

Another useful tool to investigate the roots of a polynomial over the $p$-adic field $\mathbb{Q}_{p}$ is the Newton polygon.

Definition 3.1 (Newton polygon). Let $p$ be a prime, $f=\sum_{i=0}^{n} a_{i} x^{i} \in \mathbb{Q}_{p}[X]$ be a polynomial of degree $n$, with $f(0) \neq 0$, and let $\nu_{p}$ be the $p$-adic valuation on $\mathbb{Q}_{p}$. The Newton polygon of $f$ is the set of line segments bounding the lower convex envelope of the point set $\left\{\left(i, \nu_{p}\left(a_{i}\right)\right): i \in\{0, \ldots, n\}, a_{i} \neq 0\right\} \subset \mathbb{R}^{2}$.

Number the line segments of the Newton polygon of $f$ as $S_{1}, \ldots, S_{k}$, where the slope of $S_{i+1}$ is strictly larger than the slope of $S_{i}$ (for all $i=1, \ldots, k-1$ ). Then the lengths and slopes 
3 New explicit intersective polynomials for non-solvable groups

of the $S_{i}$ yields information about the $p$-adic valuation of the roots of $f$. More precisely, the following holds (Proposition II.6.3 in [15]):

Proposition 3.2. With the above nomenclature, let $S$ be a line segment of the Newton polygon of $f$, beginning in $\left(r, \nu_{p}\left(a_{r}\right)\right)$ and ending in $\left(s, \nu_{p}\left(a_{s}\right)\right)$. Denote the slope of $S$ by $-m$. Then $f$ has precisely $s-r$ roots of $p$-adic valuation $m$.

Since the $p$-adic valuation is invariant for all roots of a given irreducible factor of $f$, Proposition 3.2 immediately yields information about the Galois group of $f$ over $\mathbb{Q}_{p}$ : its orbit lengths in the action on the roots of $f$ must be a subpartition of the partition given by the numbers $s-r$ (running through all line segments $S$ of the Newton polygon).

\subsection{The group $P S L_{3}(2)$}

We give a four-parameter polynomial $f$ leading to intersective integer polynomials $f \cdot g$ with two irreducible factors. As the "partner" polynomial $g$ would be rather complicated to write down with no parameters specialized, we only give a one-parameter version of it explicitly. The polynomial $f$ first appeared in [12].

Computations in this and the following sections were performed with the help of Magma.

Theorem 3.3. Let $a, b, c$ and $t$ be algebraically independent transcendentals over $\mathbb{Q}$. Let $f:=X^{7}-((c-2) a+2 b+c) X^{6}+\left(-(b-4)(c-1) a^{2}+\left((c-2) b^{2}+\left(2 c^{2}-5 c+4\right) b-2 c^{2}\right) a+\right.$ $\left.b\left(2 b c+2 c^{2}+b^{2}\right)\right) X^{4}+\left(\left(2 c^{2}-1\right)(b-4) a^{2}+\left(\left(-2 c^{2}+c+2\right) b^{2}+\left(5 c^{2}+2 c-4\right) b-4 c^{2}\right) a-(c+\right.$ $\left.1) b^{3}-c(2 c+3) b^{2}+c^{2} b\right) X^{3}+\left(\left(c^{2}+3 c-1\right)(4-b) a^{2}+\left((3 c-2) b^{2}-2\left(c^{2}+4 c-2\right) b+4 c^{2}\right) a+b\left(b^{2}+\right.\right.$ $\left.\left.3 b c-c^{2}\right)\right) c X^{2}+\left(2 a b c-8 a c+a b-4 a-b^{2}+2 b c\right) a c^{2} X-a^{2}(b-4) c^{3}+t X^{2}(X-c)\left(X^{2}-b X+b\right)$.

Then $f$ has Galois group $P S L_{3}(2)$ over $\mathbb{Q}(a, b, c, t)$. Furthermore, for all odd integer specializations of $a, b, c$ and $t$ preserving the Galois group, there is an irreducible polynomial $g \in \mathbb{Z}[X]$ such that specialized polynomial $f \cdot g$ has Galois group $P S L_{3}(2)$ over $\mathbb{Q}$ and has a root in every $\mathbb{Q}_{p}$.

In particular, for $a=b=c=1$, the polynomial $g:=X^{8}+(-14 t+14) X^{7}+\left(87 t^{2}-178 t+\right.$ $109) X^{6}+\left(-314 t^{3}+980 t^{2}-1210 t+431\right) X^{5}+\left(721 t^{4}-3032 t^{3}+5654 t^{2}-4119 t+1293\right) X^{4}+$ $\left(-1080 t^{5}+5700 t^{4}-14238 t^{3}+15835 t^{2}-9776 t+2542\right) X^{3}+\left(1032 t^{6}-6521 t^{5}+20397 t^{4}-\right.$ $\left.30663 t^{3}+28012 t^{2}-14338 t+3343\right) X^{2}+\left(-576 t^{7}+4212 t^{6}-15786 t^{5}+29976 t^{4}-36081 t^{3}+\right.$ $\left.27147 t^{2}-11847 t+1738\right) X+144 t^{8}-1188 t^{7}+5169 t^{6}-11874 t^{5}+17689 t^{4}-17396 t^{3}+$ $10799 t^{2}-2948 t+295 \in \mathbb{Q}(t)[X]$ fulfills the above requirements.

Proof. The polynomial $f$ already has Galois group $G:=P S L_{3}(2)$ (in its natural degree 7 


\section{New explicit intersective polynomials for non-solvable groups}

action) over $\mathbb{Q}(a, b, c, t)$ as shown in [12], Theorem 4.3. A root field of $f$ inside the Galois closure is therefore just the fixed field of a point stabilizer $U_{1}$ in this degree 7 action.

The polynomial $g$ was computed such that a root field of $g$ is the fixed field inside the Galois closure of $f$ of a maximal subgroup $U_{2}$ of index 8 in $G\left(U_{2}\right.$ is a point stabilizer of $G$, reinterpreted as $\left.P S L_{2}(7)\right)$. One checks that not only is the union of conjugates of $U_{1}$ and $U_{2}$ all of $G$, but in fact, that all metacyclic subgroups of $G$ are contained in one such conjugate, and the only solvable subgroups that are contained neither in a conjugate of $U_{1}$ nor in one of $U_{2}$ are isomorphic to $S_{4}$ or $A_{4}$.

This means that all specializations of $f \cdot g$ that preserve the Galois group have a root in every $\mathbb{Q}_{p}$ such that $p$ is (at most) tamely ramified in the Galois closure over $\mathbb{Q}$; and in fact in all $\mathbb{Q}_{p}$, as long as 2 is not wildly ramified. Now for $a, b, c, t$ all odd, $f$ is congruent modulo 2 to $X^{7}+X^{5}+X^{4}+X^{3}+1$ which is an irreducible separable polynomial over $\mathbb{F}_{2}$. This means that the splitting field of $f$ over $\mathbb{Q}_{2}$ is unramified (of degree 7 ), which completes the proof.

\subsection{The group $P \Gamma L_{2}(8)$}

From Theorem 2.2, the group $P S L_{2}(8)=P G L_{2}(8)$ has a 2-covering by subgroups $U_{1}$ of index 9 (point stabilizer in the natural action) and $U_{2}$ of index $28=\frac{8 \cdot 7}{2}$. The analagous statement remains true for $P \Gamma L_{2}(8)=P S L_{2}(8) .3$, with the subgroups $U_{1} .3$ and $U_{2} .3$. In fact, for both $P S L_{2}(8)$ and $P \Gamma L_{2}(8)$, most solvable subgroups are already contained in a conjugate of one of the two groups used for the 2-covering: the only ones that are not contained have socle $C_{7}$; more precisely, exactly $D_{7}$ (for both $P S L_{2}(8)$ and $P \Gamma L_{2}(8)$ ) and $A G L_{1}(7)$ (for $P \Gamma L_{2}(8)$ ) are not contained in any of the conjugates.

This means that any Galois extension of $\mathbb{Q}$ with group $P S L_{2}(8)$ or $P \Gamma L_{2}(8)$ will yield an intersective polynomial with two irreducible factors, just as long as we can make sure that no inertia subgroup $I_{p}$ has order divisible by 7 (for any prime $p$ ). We will first use this to generate infinitely many intersective polynomials for $P \Gamma L_{2}(8)$.

There are several known polynomials with group $P S L_{2}(8)$ or $P \Gamma L_{2}(8)$ over $\mathbb{Q}$ (see e.g. the tables in the appendix of [13]), and those can indeed be used to find intersective polynomials for both groups. Here we content ourselves with a family of intersective polynomials $f \cdot g$ for $P \Gamma L_{2}(8)$ which has a nice additional property: both $f$ and $g$ are of the form $p(x)-t q(x)$. The "intersective" property then means that there are rational functions $r_{1}(x)$ and $r_{2}(x) \in \mathbb{Q}(x)$ such that for all primes $p$, every integer value is obtained in $\mathbb{Q}_{p}$ by one of $r_{1}$ and $r_{2}$ (and even every $p$-adic integer value $t_{0} \in \mathbb{Z}_{p}$, since those can 


\section{New explicit intersective polynomials for non-solvable groups}

be approximated by rational integers and for sufficiently close approximations, the Galois groups over $\mathbb{Q}_{p}$ of the corresponding specialized polynomials are the same, by Krasner's lemma). This property is itself worth investigating in generality - a necessary condition for the existence of such pairs of rational functions for a group $G$ is the existence of a 2-covering of $G$ by subgroups $U_{1}$ and $U_{2}$ and of a class tuple in $G$ that is a genus zero tuple in both the permutation actions on $G / U_{1}$ and on $G / U_{2}$. Since these are quite restrictive conditions, a complete classification of such pairs might be achieved.

Theorem 3.4. Let $t$ be transcendental over $\mathbb{Q}, f:=\left(x^{3}+16 x^{2}+160 x+384\right)^{3}-7^{3} \cdot t\left(x^{2}+\right.$ $13 x+128)$ and

$g:=\left(x^{9}+11 x^{8}+4 x^{7}-868 x^{6}+6174 x^{5}-43974 x^{4}+37492 x^{3}-28852 x^{2}-2967 x+211\right)^{3}(x-$ $5)+2^{7} \cdot 7^{3} \cdot t\left(x^{3}-x^{2}-9 x+1\right)^{7}$. Then $f \cdot g$ has Galois group $P \Gamma L_{2}(8)$ over $\mathbb{Q}(t)$, and for every odd integer specialization $t \mapsto t_{0} \in 2 \mathbb{Z}+1$ preserving the Galois group, the specialized polynomial $f \cdot g$ has a root in every $\mathbb{Q}_{p}$.

Proof. The polynomials $f$ and $g$ parameterize coverings of the projective line $\mathbb{P}^{1}$ of degree 9 and 28 respectively, each ramified at three points, with inertia canonical invariant $(2 A, 3 A, 7 A)$, geometric monodromy group $P S L_{2}(8)$ and arithmetic monodromy group $P \Gamma L_{2}(8)$. In particular, $f$ and $g$ have the same splitting field over $\mathbb{Q}(t)$. As the class triple $(2 A, 3 A, 7 A)$ in $P \Gamma L_{2}(8)$ is a genus zero triple both in the action on 9 and on 28 points, both coverings are genus zero Belyi maps. Their computation is nowadays standard, and we only refer to [19] for further information about computation of such maps.

Let $p$ be a prime, $t_{0} \in \mathbb{Z}$, and denote the specialized polynomial of $f$, corresponding to $t \mapsto t_{0}$, by $f_{0}$. By the arguments preceding the theorem, we only have to exclude that the inertia group $I_{p}$ of $f_{0}$ at $p$ does not contain a group of order 7 . If it does, then $f_{0}$ decomposes in the form $h:=(x-a)^{7} \cdot\left(x^{2}+b x+c\right)$ over $\mathbb{F}_{p}$ (with some $a, b, c \in \mathbb{F}_{p}$ ), since $I_{p}$ has an orbit of length 7 in the action on 9 points. We solve the equation $f_{0} \equiv h \bmod p$ by computing (with Magma) the Groebner basis of the ideal generated by the coefficients of $f-h$ in $\mathbb{Z}[a, b, c, t]$. This basis contains an element $2^{11} \cdot 7$, which enforces $p \in\{2,7\}$. Now for odd $t, f$ is separable modulo 2 and therefore unramified at 2 .

For $p=7$, consider the Newton polygon corresponding to the polynomial $g(x+5)$ over $\mathbb{Q}_{7}$. The points $\left(i, \nu_{7}\left(a_{i}\right)\right)$ determining this polygon are $(0, k)$ with $k \geq 10$ (exact value depending on $\left.t_{0}\right),(1,9)$ and $(28,0)$. All other points lie on or above the line connecting $(1,9)$ and $(28,0)$. By proposition 3.2, this means that $g(x+5)$ has exactly one root of 7 -adic valuation $\geq 1$, and therefore the decomposition groups at 7 fix a root of $g$, for all specializations $t \mapsto t_{0} \in \mathbb{Z}$. This concludes the proof. 
3 New explicit intersective polynomials for non-solvable groups

\subsection{The group $M_{11}$}

The smallest Mathieu group $M_{11}$ is 2-coverable in different ways. In order to give intersective polynomials of small degree, we use a 2-covering by the two maximal subgroup of smallest index, namely the point stabilizers in the 3-transitive actions on 11 and 12 points respectively. One checks directly with a computer that the only solvable subgroups of $M_{11}$ not contained in the union of conjugates of these two subgroups are metacyclic groups $C_{3} . C_{6}$ of order 18 and several non-metacyclic groups, all of order dividing 144 .

The polynomials used in the following theorem were first found by the author in [9] (Theorem 1). For the sake of simplicity, we give rational, but not integer polynomials $g$ in the theorem below. Turning these into monic integer polynomials is trivial, but would blow up the coefficients unnecessarily.

Theorem 3.5. Let $f:=\left(x^{2}-4 x-16\right)^{5}\left(x^{2}-4 x-1\right)+2 t x^{3}(x-4)^{3}\left(x^{3}-128\right)$ and $g:=x^{11}+x^{7}(3 x+2) s+x\left(3 x^{4}+14 / 5 x^{3}+4 / 5 x^{2}-40 / 81 x-16 / 81\right) s^{2}-(x-2 / 5)(x+2 / 5) s^{3}$, where $s:=4 t / 10125$.

Then $f g$ has regular Galois group $M_{11}$ over $\mathbb{Q}(t)$, and the specialized polynomial is intersective for all specializations $t \mapsto t_{0} \in \mathbb{Z}$ that preserve the Galois group and fulfill $t_{0} \equiv 1$ $\bmod 3$.

Proof. Once again, the polynomial $g$ was computed to parameterize the stem field of an index-11 subgroup in $\operatorname{Gal}(f \mid \mathbb{Q}) \cong M_{11}$. By the argument preceding this theorem, in order to obtain intersective polynomials from a given $M_{11}$-extension, we only have to exclude wild ramification at 2 and 3 , and ramification index 3 at any prime. Since the subgroups of order 3 in $M_{11}$ have exactly three orbits of length 3 , ramification index 3 at a prime $p$ would mean that $f \equiv\left(x^{3}+a_{1} x^{2}+a_{2} x+a_{3}\right)^{3}\left(x^{3}+a_{4} x^{2}+a_{5} x+a_{6}\right) \bmod p$. Groebner basis computation as in the previous section shows that this is only possible for $p \in\{2,3,5,11\}$.

Modulo 11, $f$ has a simple zero at $x=6$ for all integer specializations of $t$, which means that the decomposition group at 11 is contained in a point stabilizer of the degree-12 action.

Modulo $2, f$ factors as $x^{10} \cdot(x-1)^{2}$, which means that the decomposition group at 2 has an orbit of length at most 2 in its action on 12 points. However, one checks directly that the orbit lengths of the "bad" candidate subgroups mentioned before the theorem are (12), $(8,4),(6,6)$ and $(6,3,3)$ respectively. Therefore the decomposition group at 2 is not one of them.

To gain information modulo 3 , set $t=1$. The polynomial $3^{12} \cdot g$ is then congruent to $2 x+2 \bmod 3$. Therefore its Newton polygon has a length-1 line segment of slope 0 ; in 


\section{References}

other words, $g$ has a linear factor over $\mathbb{Q}_{3}$.

Finally, modulo $5, f$ has at least one simple zero as long as $t$ is not divisible by 5 . Therefore, in these cases, the decomposition groups at 5 fix a point.

If $5 \mid t$, but $5^{6}$ does not divide $t$, then the Newton polygon for $f(x-3)$ shows that $f$ still has a linear factor over $\mathbb{Q}_{5}$ (the 5 -adic valuations of the coefficients $a_{i}$ of this polynomial are $\nu_{5}\left(a_{0}\right) \geq 1+\nu_{5}(t), \nu_{5}\left(a_{1}\right)=\nu_{5}(t)$, and all other valuations $\nu_{5}\left(a_{i}\right)$ strictly larger than $\nu_{5}(t)-(i-1)$. Therefore the Newton polygon contains a length-1 line segment of slope $-1)$. On the other hand, if $5^{6} \mid t$, then an analogous argument with the Newton polygon of $g$ shows that $g$ has a linear factor over $\mathbb{Q}_{5}$. This concludes the proof.

\section{References}

[1] S. Arias-de-Reyna, N. Vila, Tame Galois realizations of $G L_{2}\left(\mathbb{F}_{\ell}\right)$ over $\mathbb{Q}$. J. Number Theory 129 (2009), 1056-1065.

[2] S. Arias-de-Reyna, N. Vila, Tame Galois realizations of $G S p_{4}\left(\mathbb{F}_{\ell}\right)$ over $\mathbb{Q}$. Int. Math. Res. Not. IMRN (9) (2011), 2028-2046.

[3] R. Brandl, Integer Polynomials with Roots mod $p$ for all Primes $p$. J. Algebra 240 (2001), 822-835.

[4] D. Bubboloni, M.S. Lucido, Coverings of linear groups. Comm. Alg. 30 (5) (2002), 2143-2159.

[5] D. Bubboloni, J. Sonn, Intersective $S_{n}$ polynomials with few irreducible factors. To appear in Manuscripta Mathematica.

[6] C. Hering, Transitive linear groups and linear groups which contain irreducible subgroups of prime order. II. J. Algebra 93 (1985), 151-164.

[7] J.W. Jones, D.P. Roberts, A database of number fields. London Journal of Mathematics and Computation, 17 (1) (2014), 595-618. Database at http://hobbes.la.asu.edu/NFDB/

[8] J. Klüners, G. Malle, A database for field extensions of the rationals. LMS Journal of Computation and Mathematics 4 (2001), 182-196. Database at http://galoisdb.math.upb.de/

[9] J. König, On rational functions with monodromy group $M_{11}$. To appear in J. Symb. Comp. 


\section{References}

[10] M.J. Lavallee, B.K. Spearman, Q. Yang, Intersective polynomials with Galois group $D_{5}$. Math. J. Okayama Univ. 56, (2014), 27-33.

[11] P.D. Lee, B.K. Spearman, Q. Yang, A Parametric Family of Intersective Polynomials with Galois Group $A_{4}$. Comm. Alg. 43 (5) (2015), 1784-1790.

[12] G. Malle, Multi-parameter polynomials with given Galois group. J. Symb. Comput. 21 (2000), 1-15.

[13] G. Malle, B.H. Matzat, Inverse Galois Theory. Springer Monographs in Mathematics, Berlin-Heidelberg (1999).

[14] B. Mazur, Rational Isogenies of Prime Degree. Invent. Math. 44 (1978), 129-162.

[15] J. Neukirch, Algebraic Number Theory. Springer Verlag, Berlin-Heidelberg (1999).

[16] J. Neukirch, A. Schmidt, K. Wingberg, Cohomology of Number Fields. Springer Verlag, Berlin (2000).

[17] D. Rabayev, J. Sonn, On Galois realizations of the 2-coverable symmetric and alternating groups. Comm. Alg. 42, no. 1 (2014), 253-258.

[18] J.-P. Serre, Propriétés galoisiennes des points d'ordre fini des courbes elliptiques. Inventiones math. 15 (1972), 259-331.

[19] J. Sijsling, J. Voight, On computing Belyi maps. Publications mathématiques de Besancon (2014), 73-131.

[20] J. Sonn, Polynomials with roots in $\mathbb{Q}_{p}$ for all p. Proc. Americ. Math. Soc. 136 (2008), no.6, 1955-1960.

[21] J. Sonn, Two remarks on the inverse Galois problem for intersective polynomials. J. Theor. Nombres Bordeaux, 21 (2009), no. 2, 437-439. 